\title{
Devolatilization of Polymers by Diffusion and Foaming
}

\author{
INGO GeSTRING* AND DieTER MeweS \\ Institute for Process Engineering, University of Hannover, \\ Callinstr. 36, D-30167 Hannover, Germany
}

\begin{abstract}
Devolatilization is a key step in polymer processing. Low-molecularweight components are removed from a polymeric system. The transport of these components takes place by diffusion to the polymer-vapor interface. This interface can be formed by free surfaces of single-phase polymer melts or by bubbles. In this study the transport with and without bubble nucleation is investigated independently from each other in a specially designed apparatus.

The mass transport in thin films and in rotating pools with surface renewal is measured. High surface renewal rates and thick films enhance the mass transfer for single phase flow. For two phase flow and devolatilization the conditions for bubble nucleation and foam formation are investigated. The bubble nucleation is observed in the rotating pool in the area of high shear velocity.
\end{abstract}

\section{INTRODUCTION}

D EVOLATILIZATION OF POLYMERS is a separation process. After the polymerization of unreacted monomers, solvents or impurities remain in the polymer. These components must be removed in order to obtain specific material properties with zero or very low monomer concentration. The devolatilization step during polymer processing often requires expensive equipment. Therefore it is necessary to have a detailed knowledge about the devolatilization process in order to reduce costs.

The concentration range of the volatiles, which have to be removed, is from several ppm to several tens of a percent. A large variety of equipment is used for the devolatilization step. This equipment can be classified into two main categories: rotating devolatilizers like the vented extruder or thin film evaporator and still devolatilizer or the flash evaporator.

This revised paper was presented in its original form at the 58th Annual Technical Conference (ANTEC 2000) held in Orlando, FL, May 7-11, 2000 and the copyright is held by the Society of Plastics Engineers.

*Author to whom correspondence should be addressed. E-mail: gestring@c36.uni-hannover.de

Journal of REINFORCED PlAstiCs AND COMPOSITES, Vol. 21, No. 5/2002 
Two dominant mass transfer mechanisms exist. On the one hand a diffusion process takes place due to a concentration gradient close to the exposed polymer surface. In rotating devolatilizers the mass transfer occurs at the polymer-vapor interface of the rotating pools in front of scrapers or wipes in thin films. The surface of the pool and the film is permanently renewed. The result of the convective transport in pool and film is an increase in the concentration gradient and by this the process efficiency is enhanced. On the other hand bubbles of the volatile component are nucleated under specific conditions in the polymer. The bubbles grow, coalesce and reach the polymer-vapor interface, where they rupture and release the volatiles to the vapor phase. This process is known as foam devolatilization. Because of the large internal surface area of the foam, this kind of devolatilization process is more efficient than a simple diffusive transport.

\section{STATEMENT OF THEORY AND DEFINITIONS}

\section{Diffusing Film Model}

Using the penetration theory [1] is the most popular way to calculate the bubble free mass transfer rate in vented extruders or similar equipment. The penetration model assumes the films to be semi-infinite media. The average mass flow rate $\dot{M}_{f}$ over the exposure time of the film $t_{f}$ is given by

$$
\frac{\dot{M}_{f}}{A_{f}}=\frac{1}{t_{f}} \int_{0}^{t_{f}} \dot{m}_{f} d t=2 \sqrt{\frac{D}{\pi t_{f}}} \rho\left(\xi-\xi_{e q}\right)
$$

with $\dot{m}_{f}$ as the instantaneous mass flow rate per area, $A_{f}$ as the interfacial surface area, $t_{f}$ as the renewal time of the film, $D$ as the diffusion coefficient, $\rho$ as the melt liquid density, $\xi$ as the volatile weight fraction of the polymer melt and $\xi_{e q}$ as the equilibrium weight fraction of the polymer-vapor interface. From Equation (1) the mass transfer coefficient $k_{f}$ is given by

$$
k_{f}=2 \sqrt{\frac{D}{\pi t_{f}}}
$$

Collins et al. [2] calculate mass transfer coefficients for bubble free mass transfer in fully intermeshing, corotating twin-screw extruders. The experimental measured values of the mass transfer coefficient are smaller than the calculated ones based on penetration theory. They conclude that the surface area for mass transfer is substantially smaller than the value computed on the basis that continuous films are formed on the surfaces of the screws and the barrel wall. 


\section{Bubble Nucleation and Foam Formation}

The necessary condition for creating bubbles by nucleation in a polymer melt is that the mixture of the volatile component and the polymer melt is superheated. The vapor pressure of the volatile component $p_{v}$ must exceed the ambient pressure $p$. The degree of superheat is given by

$$
\Delta p_{s}=p_{v}-p
$$

Biesenberger and Lee [3] conclude from their experimental results that superheat alone is not sufficient for bubble nucleation in polymer melts. Shear forces are also essential. They assume that heterogeneous nucleation sites exist in microscopic cavities. Favelukis et al. [4] give results for the nucleation and growth of bubbles in a Couette apparatus. They postulate that a critical shear rate for bubble nucleation exists. Han and Han [5] observe bubble formation in a shear flow field even at an unsaturated condition.

\section{DESCRIPTION OF EQUIPMENT AND PROCESSES}

A specially designed apparatus is used for the experimental study. A photograph of the equipment is shown in Figure 1. A drum rotates around a fixed inner barrel. In front of a blade, a rolling pool is created. Due to a small space between the blade

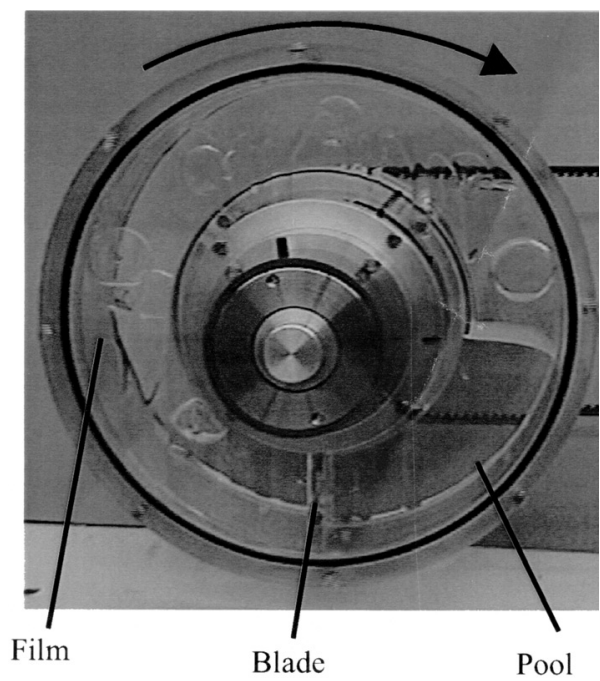

Figure 1. Photography of the rotating drum. 


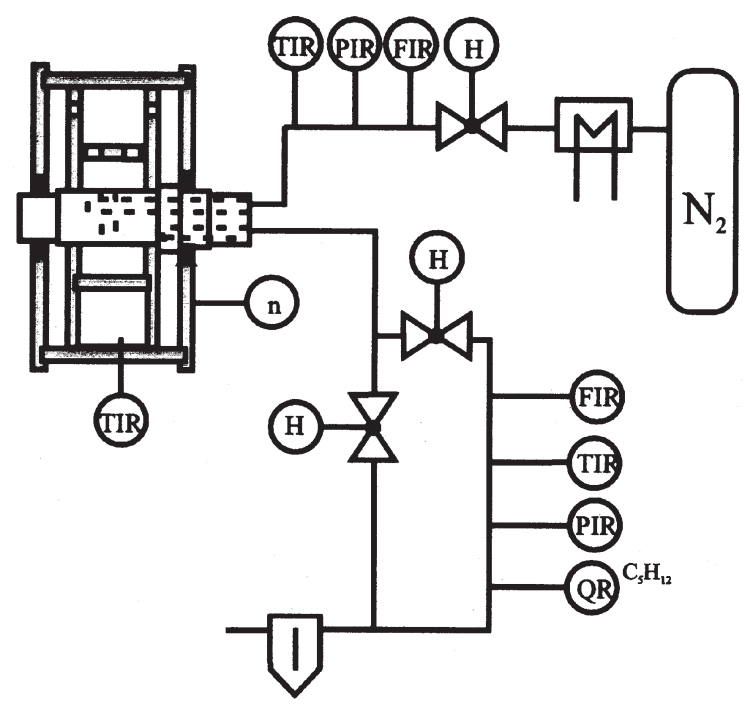

Figure 2. Experimental setup.

and the drum, a thin film is formed at the inner surface of the drum. The ratio of the inner and the outer cylinder is 1.7 , which is similar to the ratio of the barrel and the screw of a devolatilization extruder.

The polymer matrix is polydimethylsiloxane with viscosities between 1 and 100 $\mathrm{Pa}$ s; $n$-pentane is used as the volatile component. The volatile concentration ranged from 1 to 7 weight percent.

The bubble-free devolatilization is studied under atmospheric conditions by pumping nitrogen through the drum. The experimental setup is shown in Figure 2. The mass flow of nitrogen and the concentration of $n$-pentane in nitrogen are measured. With this information the mass flow of $n$-pentane and the degree of separation can be calculated. The mass transfer of the film and the pool can be measured independently of each other.

The bubble nucleation and foam formation experiments are done under pressure at vacuum. After vacuum is applied, the rotation of the drum starts. The nucleation sites and the foam formation and heights are videotaped.

\section{RESULTS AND DISCUSSION}

\section{Mass Transfer}

In Figure 3 the mass flow of the film is shown for different rotational speeds as a function of dimensionless time given by the Fourier-number. 


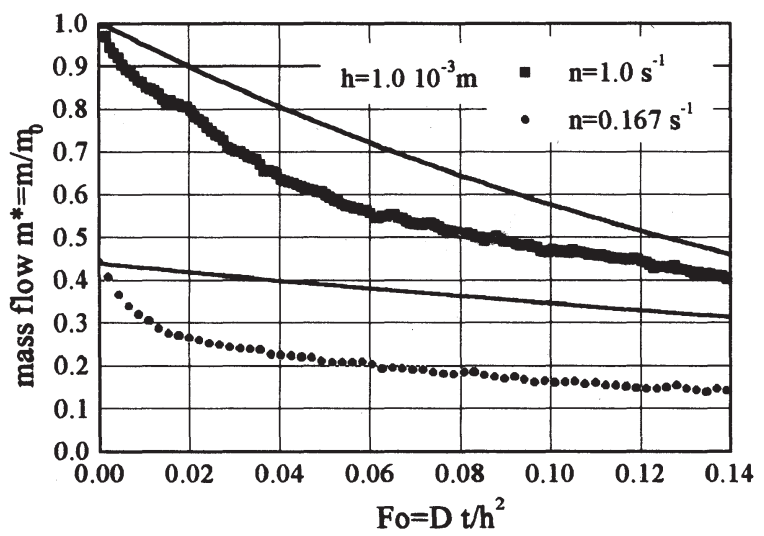

Figure 3. Measured and calculated mass flow for diffusive transport of the film.

The mass flow is similar to an exponential decay. For small Fourier-numbers the ratio of the mass flows

$$
\frac{\dot{m}_{1}}{\dot{m}_{2}} \sim \sqrt{\frac{n_{1}}{n_{2}}}
$$

is proportional to the square root of the ratio of the rotational speed $n$ as supposed by the penetration theory. But for large Fo-numbers this model fails to calculate the mass transfer correctly. The measured values are smaller than the calculated ones. By using the penetration theory it is assumed that film and pool are mixed completely after every turn of the drum and that the concentration of $n$-pentane in the pool is homogeneous.

The penetration theory does not consider the convective term in the molecular mass transport balance equation. For constant diffusion coefficients and incompressible fluids in two dimensions this equation is given by

$$
\frac{\partial c}{\partial t}+\left(v_{x} \frac{\partial c}{\partial x}+v_{y} \frac{\partial c}{\partial y}\right)=D\left(\frac{\partial^{2} c}{\partial x^{2}}+\frac{\partial^{2} c}{\partial y^{2}}\right)
$$

A CFD-simulation is done in order to obtain the concentration distribution in the pool by using Equation (6). In Figure 4 the concentration field of the pool for the mass transfer of the thin film is shown. The highest concentration is in the corner of the blade and the inner cylinder. In the middle of the pool, where the convective flow is small, the concentrations are higher than at the edges of the pool. In contrast to the pool the concentrations of the film are low. Therefore the 


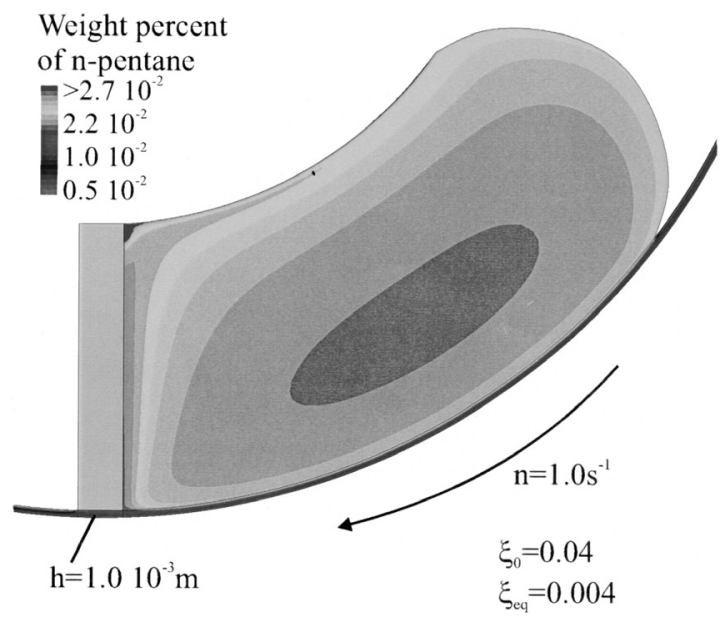

Figure 4. Concentration profile of the pool after $400 \mathrm{~s}$ for diffusive transport of the film.

convective parts of Equation (5) must be considered for the devolatilization process of polymers by diffusion.

In the experimental investigations it is shown that thicker films enhance the mass transfer. Thick films result in a better mixing of polymer in the film and the pool. A thick film leaves the pool with a higher concentration than a thin film. The viscosity of the polymer has only a small impact on the mass transfer. By a better mixing of pool and film the efficiency of the process could be enhanced.

\section{Foam Devolatilization}

For studying the bubble nucleation and the foam formation process, the polymer melt is carefully filled into the drum, avoiding any air bubbles in the melt. These bubbles are potential nucleation sites. The vapor pressure of $n$-pentane in polydimethylsiloxane is calculated with the Flory-Huggins equation [6]. By varying the concentration of $n$-pentane and the pressure level, different degrees of superheat can be applied. If vacuum pressures are applied to the drum without rotation no bubbles can be observed. The degree of superheat is varied between $20 \times 10^{2}$ and $500 \times 10^{2} \mathrm{~Pa}$. After the rotation starts, bubbles are nucleated immediately in the area of high deformation rate. Therefore superheat and deformation rate are two essential conditions for bubble nucleation in polymeric liquids.

The highest deformation rate is next to the outer cylinder. The degree of superheat and the shear velocity are given in Figure 5 for different rotational speeds. The highest shear velocity is observed in front of the blade, but the lowest degree of superheat is there also. Therefore bubble nucleation only can take place in the area 


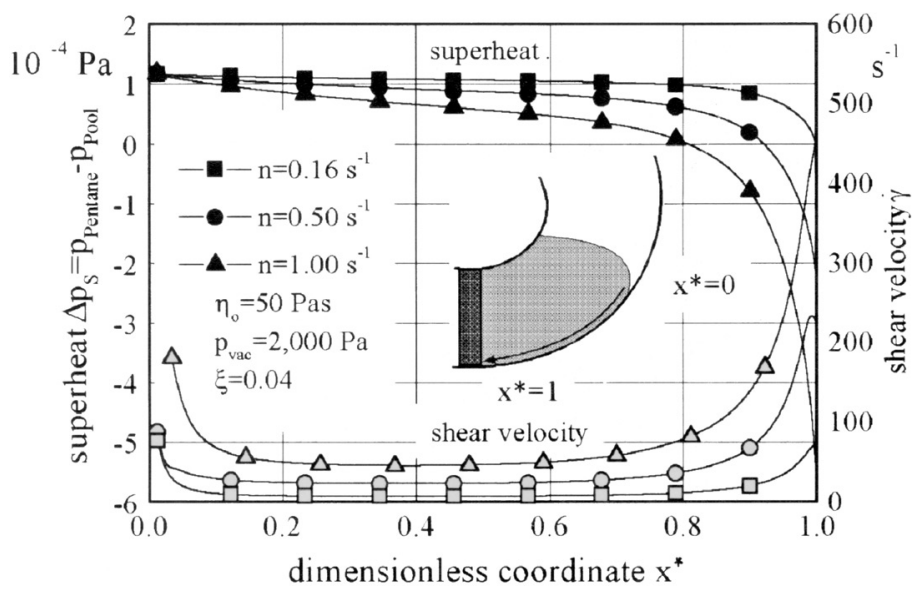

Figure 5. Shear velocity and degree of superheat at the inside of the drum.

of the outer cylinder next to the free surface. This coincides with the visual observations. The different phases of the foam formation are shown in Figure 6. After the bubble nucleation has taken place, the bubbles are transported to the blade into a region of higher pressure where they shrink. As the bubbles are transported by the rotational movement to a region of lower pressure, the bubbles grow, coalesce and form a foam. The streamlines and the pressure distribution of the pool is shown in Figure 7.

The initial bubble nucleation and roam formation are rapid passing processes. The maximum foam expansion is reached several seconds after the rotation starts. High rotational speeds and high concentrations of the volatile component accelerate the foam formation. The foam expansion is shown in Figure 8. After the foam

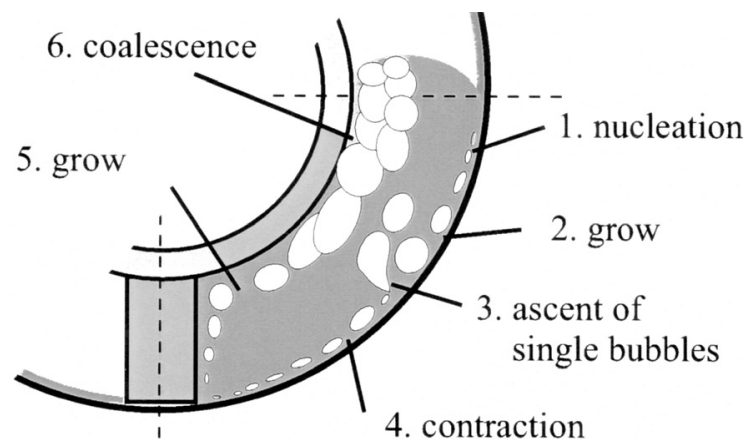

Figure 6. Different phases of foam formation 


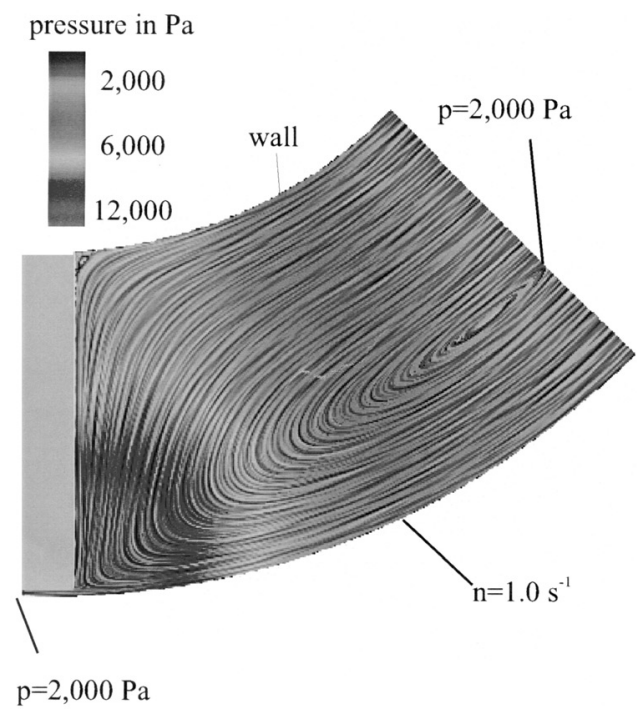

Figure 7. Texture of the flow and pressure field.

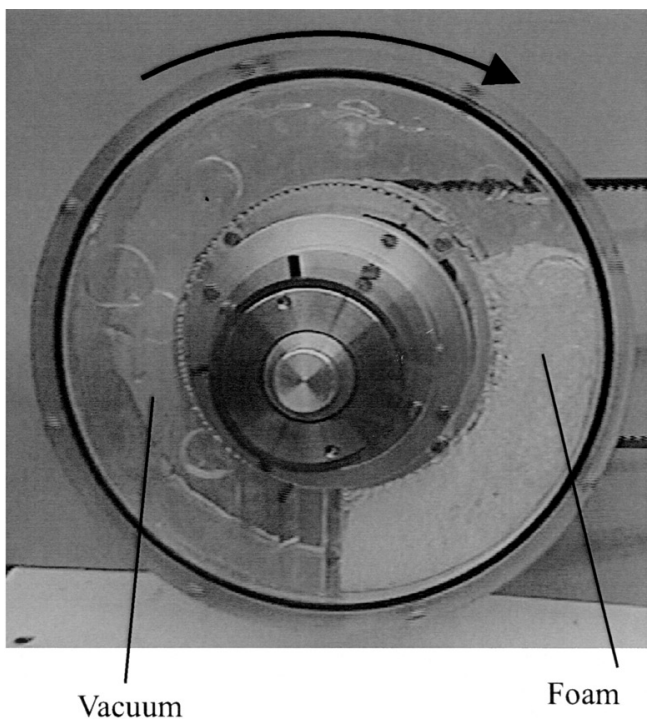

Figure 8. Photograph of the rotating drum with foam. 
has reached its maximum volume it depends mainly on the viscosity and the rotational speed how long the polymer melt foams. High viscosities and high rotational speeds suppress the growth of bubbles. If the initial foam formation is followed by a decreasing rotational speed the bubbles stay longer in the polymer melt. These bubbles are transported with the motion of the pool from the outer cylinder via the blade and from the inner cylinder to the surface of the pool. The center of the pool volume is not affected by these bubbles.

\section{CONCLUSIONS}

Devolatilization of polymers without bubble nucleation is a slow process. Although the concentration gradient at the free surface is high because of the renewal of the surface, the laminar rotational flow of the melt in the pool prevents a good blending of the polymer. The edges of the polymer melt inside the pool and within the thin film may be quite well degassed. There may still remain a higher concentration inside the pool. By better mixing the devolatilization efficiency can be enhanced.

For devolatilization by foam the bubble nucleation is accelerated in the beginning by a high shear rate. After the initial flash the high rotational speed of the pool prevents bubbles from growing.

\section{NOMENCLATURE}

$A_{f}$ interfacial surface area

$c$ concentration

$D$ diffusion coefficient

$F_{0}$ Fourier-number

$h$ film height

$k_{f}$ mass transfer coefficient

$n$ rotational speed

$\dot{m}_{f}$ mass flow per area

$p$ ambient pressure

$p_{v}$ vapor pressure of the volatile

$t$ time

$t_{f}$ renewal rate of the film

$\xi$ weight fraction of the volatile component

$\xi_{\text {eq }}$ weight fraction of the volatile component at the interface

\section{ACKOWLEDGEMENTS}

The authors would like to gratefully acknowledge the financial support from the 
AiF (Arbeitskreis industrieller Forschungsvereinigungen e.V.) and the GVT (Forschungs-Gesellschaft Verfahrenstechnik e.V.).

\section{REFERENCES}

1. Crank, J., The Mathematics of Diffusion, Oxford University Press, London (1956).

2. Collins, G. P., Denson, C. D. and Astrarita, G., Determination of Mass Transfer Coefficients for Bubble-Free Devolatilization of Polymeric Solutions in Twin-Screw, AIChE Journal, 31:8 1288-1296 (1985).

3. Biesenberger, J. A. and Lee, S. T., A Fundamental Study of Polymer Melt Devolatilization: I. Some Experiments on Foam Enhanced Devolatilization, Poly. Eng. Sc., 26:982 (1986).

4. Favelukis, M., Tadmor, Z. and Semiat, R., Bubble Growth in a Viscous Liquid in a Simple Shear Flow, AIChE Journal, 45:4 691-695 (1999).

5. Han, J. H. and Han, Ch. D., A Study of Bubble Nucleation in a Mixture of Molten Polymer and Volatile Liquid in a Shear Flow Field, Polym. Eng. Sc., 28:24 1616-1627 (1988).

6. Flory, P. J., Principles of Polymer Chemistry, Cornell University Press, Ithaca, New York (1953). 